\title{
A SECURITY MODEL FOR ANONYMOUS CREDENTIAL SYSTEMS
}

\author{
Andreas Pashalidis* and Chris J. Mitchell \\ Information Security Group, Royal Holloway, University of London \\ \{ A.Pashalidis,C.Mitchell \}@ rhul.ac.uk
}

\begin{abstract}
This paper proposes a formal model of the Bellare-Rogaway type [Bellare and Rogaway, 1994] that enables one to prove the security of an anonymous credential system in a complexity theoretic framework. The model abstracts away from how a specific instance of anonymous credential system achieves its goals; instead it defines what these goals are. The notions of credential unforgeability, non-transferability, pseudonym unlinkability and pseudonym owner protection are formally defined and the relationships between them are explored. The model is a step towards a formal treatment of the level of privacy protection that anonymous credential systems can and should achieve, both in terms of pseudonym unlinkability and user anonymity.
\end{abstract}

Keywords: anonymous credential systems, pseudonym systems, privacy, anonymity, unlinkability, provable security

\section{INTRODUCTION}

\subsection{Background and motivation}

Anonymous credential or 'pseudonym' systems allow users to interact with organisations using distinct and unlinkable pseudonyms. In particular, a user can obtain a credential (a statement of a designated type that attests to one or more of the user's attributes) from one organisation and then 'show' it to another, such that the two organisations cannot link the issuing and showing acts; this renders the user's transactions unlinkable. Of course this unlinkability is limited; if only one credential is ever issued with a particular set of attributes, then clearly all credential showings containing this set of attributes can be linked

*The author is sponsored by the State Scholarship Foundation of Greece. 
to each other and to the unique issued credential. Pseudonym systems must prevent users from showing credentials that have not been issued (i.e. they must guarantee 'credential unforgeability'), and prevent users from pooling their credentials (for example, to collectively obtain a new credential that each user individually would not be able to). This latter property is usually referred to as 'credential non-transferability'.

Security models of pseudonym systems, and proofs (where given), do not usually allow reasoning about the resulting degrees of user anonymity and pseudonym unlinkability. This paper, following the ideas first set out by Bellare and Rogaway in [Bellare and Rogaway, 1994], proposes a model that is based on complexity theoretic arguments and which potentially leads to information theoretic anonymity metrics. It abstracts away from the particulars of how specific pseudonym system instances achieve their goals; instead it focuses on what these goals are. The model captures security properties for both organisations (credential unforgeability and non-transferability), and users, both in terms of 'traditional' security (pseudonym owner protection) and privacy (pseudonym unlinkability and user anonymity). The model makes a clear distinction between the different notions and allows the relationships between them to be analysed.

\section{$1.2 \quad$ Related work}

Pseudonym systems were first introduced by Chaum in the 1980s [Chaum, 1985]. Since then, numerous pseudonym systems have been proposed, each with its own particular set of entities, underlying problems, assumptions and properties. Some examples are given in [Brands, 2000; Camenisch and Lysyanskaya, 2001; Chaum and Evertse, 1987; Damgard, 1990]. The most relevant work to this paper is probably the formal treatment of the anonymous credential system in [Camenisch and Lysyanskaya, 2001]. There, security is defined based on the indistinguishability between the transcripts of protocols that occur in an 'ideal' world (where a universally trusted party guarantees security), and the 'real world' (where such a party does not exist). In that model, transactions between users and organisations correspond to well-defined events, and the adversary acts like an event scheduler; he can arbitrarily trigger events of his choice. In the model of [Camenisch and Lysyanskaya, 2001], however, the relationship between the different security notions that a pseudonym system should satisfy is somewhat hidden by the fact that the universally trusted party takes care of them. Also, in that model, the adversary is not allowed to corrupt players in an adaptive fashion. While our model retains the property that the adversary gets to specify 
the order of events in the system, he can also adaptively corrupt players. Further, the model allows a relatively easy analysis of the relationships between different notions. This is due to the fact that we abstract away from properties that do not lie at the same level of abstraction as that at which a pseudonym system operates.

\subsection{What we don't do}

Our model does not capture 'traditional' communications security properties, such as entity authentication. This is not an omission; these issues are outside the scope of the model (other well-established security models can be used to reason about such issues). Of course, if users do not authenticate organisations, and if the integrity and confidentiality of communications in the system are not guaranteed at the session level, then there cannot be any security. However, the way these services are provided lies at a different level of abstraction. We therefore assume that they are provided by the infrastructure that allows users and organisations to communicate. We also assume that, within this infrastructure, users remain anonymous to organisations (i.e. we assume an anonymous channel).

The remainder of the paper is organised as follows. The next section describes the formal model of pseudonym systems. Section 2.2 establishes the notions of pseudonym owner protection, credential unforgeability and credential non-transferability, which together capture the notions of soundness for a scheme. Further, section 2.3 provides a brief discussion of the notions and explains the relationships between them. Section 2.4 establishes the notion of pseudonym unlinkability which is discussed in section 2.5. Further, section 2.6 establishes the notion of pseudonym indistinguishability and shows it is a necessary condition for unlinkability. Finally, section 2.7 addresses the issue of anonymity in pseudonym systems, while section 3 concludes the paper and gives directions for further research.

\section{SECURITY OF PSEUDONYM SYSTEMS}

In this section we describe our model of a pseudonym system. We regard a pseudonym system as being comprised of the players in the system and the procedures through which they interact. The players, in particular, are divided into users, issuing organisations and verifying organisations. Since users are known to each organisation under a different pseudonym, indeed possibly under multiple pseudonyms, a procedure must be in place according to which a user and an organisation establish a new pseudonym; we call this the 'pseudonym establishment 
protocol'. Procedures must also be in place that allow users to obtain credentials (on the pseudonym that was established with the issuer) and to show them (on the pseudonym that was established with the verifier). We call the former the 'credential issuing protocol' and the latter the 'credential showing protocol'.

In our model, credential types are in one-to-one correspondence with (combinations of) user attributes; in other words, each combination of attributes defines a credential type. An organisation, for example, that issues demographic credentials containing the fields sex and age group, with possible values of $\{$ male, female $\}$ and $\{18-, 18-30,30-50,50+\}$ respectively, in our model may actually issue up to 8 different credential types (one for each combination of values).

\subsection{The model}

A protocol prot is assumed to be a tuple of interactive Turing machines; an execution of prot is said to be successful if and only if all machines accept. The set of all non-zero polynomial functions in the natural number $k$ is denoted by poly $(k)$. A real-valued function $\epsilon: \mathbb{N} \rightarrow \mathbb{R}$, is said to be negligible in $k$ if and only if $0 \leq \epsilon(k)<1 /|q(k)|$ for any $q \in \operatorname{poly}(k)$ and for all sufficiently large $k$.

REMARK 1 We are concerned in this paper with situations where two functions $f$ and $g$ satisfy $f(k)>g(k)+\epsilon(k)$ for any negligible function $\epsilon$ and for all sufficiently large $k$. To simplify the discussion we abuse our notation slightly and simply say that $f$ is greater than $g+\epsilon(k)$, i.e. we omit explicit references to $k$, and we also omit the rider 'for all sufficiently large $k$ '.

DEFINITION 1 A pseudonym system is a tuple

$$
\text { ( } k \text {, init, } U, I, V, P, T \text {, peprot, ciprot, csprot) }
$$

whose elements are as follows.

- $k$ (a natural number) is the system security parameter.

- init is the initialisation algorithm; on input $k$, it outputs the elements of the sets $U, I, V$ and descriptions of the sets $P, T$. Hence, $U, I, V$ (and also $P$ and $T$ ) are (implicitly) regarded as functions of $k$.

- $U$ is the set of users, $|U| \in \operatorname{poly}(k)$.

- I is the set of credential issuing organisations ('issuers' in short), $|I| \in \operatorname{poly}(k)$. 
- $V$ is the set of credential verifying organisations ('verifiers' in short), $|V| \in \operatorname{poly}(k)$.

- $P$ is the set of pseudonyms.

- $T$ is the set of credential types.

- peprot is the pseudonym establishment protocol: any user/organisation pair $(u, o) \in U \times(I \cup V)$ may execute peprot; if the protocol succeeds, $u$ and $o$ will have established a pseudonym $p \in P$ and we

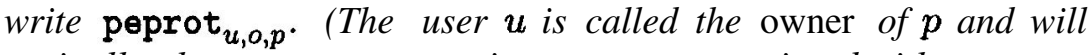
typically also possess some private output associated with $p$ as necessary to engage in ciprot and csprot.)

- ciprot is the credential issuing protocol: any user/issuer pair $(u, i) \in U \times I$ may execute ciprot with respect to a pseudonym $p \in P$ associated with $u$ and $i$ (established using peprot) and for a particular credential type $t \in T$. If successful, we say that $i$ has issued a credential of type $t$ on pseudonym $p$ to $u$, and we write ciprot $_{i, p, t}$.

- csprot is the credential showing protocol: any user/verifier pair $(u, v) \in U \times V$ may execute csprot with respect to a pseudonym $p \in P$ associated with $u$ and $v$ (established using peprot) and for a particular credential type $t \in T$; if the protocol succeeds we say that $u$ has shown a credential of type $t$ on pseudonym $p$ to $s$ and we write csprot $_{v, p, t}$.

Each issuer $i \in I$ defines a set $T_{i} \subseteq T$ of credential types that it intends to issue in the future ${ }^{1}$. It is required that, for all distinct $i, i^{\prime} \in I$, $T_{i} \cap T_{i^{\prime}}=\emptyset^{2}$. We denote the set of active credential types in the system by $T^{*} \stackrel{\text { def }}{=} \bigcup_{i \in I} T_{i}$. It holds that $\left|T^{*}\right| \in \operatorname{poly}(k)$.

\subsection{The games and soundness}

In order to formalise our notions of security for a pseudonym system, we define a series of games between two Turing machines: a Challenger and an Adversary. Each game captures a specific property of the pseudonym system. In this section we define Game 1, which captures 'pseudonym owner protection', Game 2, which captures 'credential unforgeability', and Game 3, which captures 'credential non-transferability'. In sections 2.4 and 2.6 below we define Game 4 and Game 5, which capture 'unlinkability' and 'indistinguishability' of pseudonyms, respectively. 
At the beginning of all games, the Challenger sets up the system by running init. At this point, the Challenger controls all users, issuers and verifiers of the system. He defines the sets $T_{i}$ for each issuer. The Adversary, which is assumed to be a probabilistic polynomial time (and space) algorithm and is denoted by $\mathcal{A}$, then receives as input the sets $U$, $I, V$, and $T_{i}$, descriptions of the sets $P$ and $T$, and the system's public information. As explained above, it is assumed that the underlying communication infrastructure provides authentication of issuers and verifiers to users, that it protects the integrity and confidentiality of their communications, and that it binds each protocol execution to exactly one session between the involved parties. Thus, $\mathcal{A}$ models a passive adversary that faithfully transmits messages between parties.

Each of the games consists of two distinct and successive phases. During the first phase of each game, $\mathcal{A}$ may issue (oracle type) queries to the Challenger; during the second phase he may not. During the first phase of Game 1, 2 and 3, $\mathcal{A}$ may issue the following types of query to the Challenger.

runpeprot $(u, o): \mathcal{A}$ may arbitrarily select a user/organisation pair $(u, o) \in U \times(I \cup V)$ and issue this query. When this happens, the Challenger makes $u$ and $o$ execute peprot pu,o,p $_{\text {. The }}$ Challenger replies true if the protocol execution is successful and false otherwise. (If the execution is successful, $u$ and $o$ will have established a new pseudonym $p \in P ; \mathcal{A}$, however, does not learn its value.)

runciprot $(u, i, t): \mathcal{A}$ may arbitrarily select a user/issuer pair $(u, i) \in$ $U \times I$ and a credential type $t \in T_{i}$ and issue this query. When this happens, the Challenger selects a pseudonym $p$ from set of pseudonyms that $u$ and $i$ have established ${ }^{3}$ and makes $u$ and $i$ execute ciprot $_{i, p, t}$. He replies true if the protocol execution is successful and false otherwise (including the case where $u$ and $i$ have not established any pseudonym). Note that $\mathcal{A}$ does not learn the value of $p$.

runcsprot $(u, v, t): \mathcal{A}$ may arbitrarily select a user/verifier pair $(u, v) \in$ $U \times V$ and a credential type $t \in T$ and issue this query. When this happens, the Challenger selects a pseudonym $p$ from the set of pseudonyms that $u$ and $v$ have established and makes $u$ and $v$ execute $\operatorname{csprot}_{v, p, t}$. He replies true if the protocol execution is successful and false otherwise (including the case where $u$ and $v$ have not established any pseudonym). Note that $\mathcal{A}$ does not learn the value of $p$.

corruptUser $(u): \mathcal{A}$ may arbitrarily select a user $u \in U$ and issue this query. When this happens, the Challenger hands all the private information of $u$ to $\mathcal{A}$. This includes $u$ 's pseudonyms, credentials and all his past protocol views. From that point on, the control of $u$ is passed from the Challenger to $\mathcal{A}$. 
corruptIssuer $(i): \mathcal{A}$ may arbitrarily select an issuer $i \in I$ and issue this query. When this happens, the Challenger hands all the private information of $i$ to $\mathcal{A}$. This includes the set of pseudonyms $i$ has established and all its past protocol views. From that point on, the control of $i$ is passed from the Challenger to $\mathcal{A}$.

corruptVerifier $(v): \mathcal{A}$ may arbitrarily select a verifier $v \in V$ and issue this query. When this happens, the Challenger hands all the private information of $v$ to $\mathcal{A}$. This includes the set of pseudonyms $v$ has established and all its past protocol views. From that point on, the control of $v$ is passed from the Challenger to $\mathcal{A}$.

In all games, a global and monotonically increasing variable $\tau$ counts $\mathcal{A}$ 's queries. We say that the query is issued at the time indicated by $\tau$. At some point in time, $\mathcal{A}$ exits the first phase and enters the second phase. The value of $\tau$ at that point is denoted by $\tau_{\max }$. In the second phase $\mathcal{A}$ may no longer issue any queries; what happens is specific to each game and is described below.

To describe the games we require some additional notation. In the following, $P_{u, o} \subseteq P$ denotes the set of pseudonyms the user $u \in U$ has established with the organisation $o \in(I \cup V)$ at time $\tau_{\max }$ (via $\mathcal{A}$ 's peprot

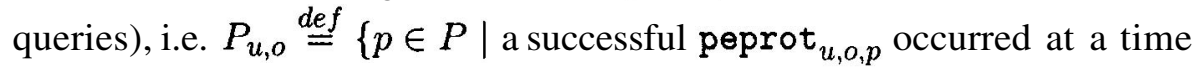
$\left.\tau \leq \tau_{\max }\right\}$. The set of pseudonyms belonging to $u$ is defined as $P_{u} \stackrel{\text { def }}{=}$ $\bigcup_{o \in(I \cup V)} P_{u, o}$ and the set of pseudonyms that $o$ has established is defined as $P_{o} \stackrel{\text { def }}{=} \bigcup_{u \in U} P_{u, o}$. (Since $\mathcal{A}$ does not learn the value of pseudonyms during their establishment, only $u$ knows $P_{u}$ and only $o$ knows $P_{o}$.) The set of active pseudonyms in the system is defined as $P^{*} \stackrel{\text { def }}{=} \bigcup_{u \in U} P_{u}$, or, equivalently, $P^{*} \stackrel{\text { def }}{=} \bigcup_{o \in(I \cup V)} P_{o}$. Since $\mathcal{A}$ is polynomially bounded in $k$, it holds that $\left|P^{*}\right| \in \operatorname{poly}(k)$. It is required that, for all distinct $u, u^{\prime} \in U, P_{u} \cap P_{u^{\prime}}=\emptyset^{4}$. The function $f: P^{*} \rightarrow U$ maps pseudonyms to their owners, which is well-defined by the assumption that $P_{u} \cap P_{u^{\prime}}=\emptyset$.

Let $\hat{U} \subseteq U, \hat{I} \subseteq I$ and $\hat{V} \subseteq V$ denote the subsets of users, issuers and verifiers respectively that $\mathcal{A}$ corrupted during the first phase. Further, let $P_{u, t}(x) \subseteq P_{u}$ denote the subset of pseudonyms belonging to user $u \in U$ on which a credential of type $t \in T^{*}$ has been issued prior to time $x$, i.e. $P_{u, t}(x) \stackrel{\text { def }}{=}\left\{p \in P_{u} \mid\right.$ a successful ciprot ${ }_{, p, t}$ occurred at time $\tau \leq x\}$.

We now describe the second phase of Games 1, 2 and 3. As mentioned above, $\mathcal{A}$ may no longer issue queries to the Challenger in this phase. He may, however, engage in $\operatorname{ciprot}_{p, i, t}$ and $\operatorname{csprot}_{p, v, t}$ executions directly with organisations (while pretending to be the user $f(p)$ ). 
GAME 1 (pseudonym owner protection): $\mathcal{A}$ selects a pseudonym/verifier/type triple $(p, v, t) \in P^{*} \times(V-\hat{V}) \times T$ such that $f(p) \in(U-\hat{U})$. We say that $\mathcal{A}$ wins the game iff he can make $v$ accept in a $\operatorname{csprot}_{p, v, t}$ execution with probability greater than any negligible function in $k$.

GAME 2 (credential unforgeability): $\mathcal{A}$ selects a pseudonym/verifier/type triple $(p, v, t) \in P^{*} \times(V-\hat{V}) \times\left(T-\bigcup_{i \in \hat{I}} T_{i}\right)$ such that $P_{f(p), t}\left(\tau_{\max }\right)=\emptyset$ and $\bigcup_{u \in \hat{U}} P_{u, t}\left(\tau_{\max }\right)=\emptyset$. We say that $\mathcal{A}$ wins the game iff he can make $v$ accept in a csprot $_{p, v, t}$ execution with probability greater than any negligible function in $k$.

GAME 3 (credential non-transferability): $\mathcal{A}$ selects a pseudonym/verifier/type triple $(p, v, t) \in P^{*} \times(V-\hat{V}) \times\left(T-\bigcup_{i \in \hat{I}} T_{i}\right)$ such that $P_{f(p), t}\left(\tau_{\max }\right)=\emptyset$. We say that $\mathcal{A}$ wins the game iff he can make $v$ accept in a $\operatorname{csprot}_{v, p, t}$ execution with probability greater than any negligible function in $k$.

DEFINITION 2 A pseudonym system is said to offer pseudonym owner protection, credential unforgeability or credential non-transferability if and only if no adversary $\mathcal{A}$ can win Game 1, 2 or 3, respectively.

\subsection{Discussion}

Game 1, 'pseudonym owner protection', captures security for users; nobody - even when colluding with users, issuers and verifiers - should be able to successfully show a credential on a pseudonym of which he is not the owner (i.e. on a pseudonym which was not established by himself). The property is typically achieved by having the pseudonym establishment protocol generate some private output for the user. This output is then treated as a secret that enables the user to authenticate himself as the pseudonym owner during the execution of the credential issuing and showing protocols.

Games 2 and 3 capture security for organisations. In particular, Game 2 captures what is usually perceived as 'credential unforgeability'. If a (dishonest) user can construct a credential by himself (i.e. without obtaining it legitimately from an issuing organisation), if, in other words, the user can forge the credential, then the system clearly does not offer credential unforgeability. Game 2 captures unforgeability in this sense. There is, however, a simplistic way for a user to 'forge' a credential: by 'borrowing' it from another user with whom he colludes (and who legitimately obtained the credential from an issuing organisation). This type of 'forgery' is not captured by Game 2. In some applications credential sharing is not a concern while forgery is.

Game 3, credential non-transferability, captures the case of credential sharing between users. In a system that offers credential non- 
transferability, no user can successfully show a credential of a type he himself was never issued. This holds even in the case he colludes with other users that have been issued credentials of that type.

It is interesting to observe the relationship between the notions of unforgeability and non-transferability: the latter, being stronger, implies the former. Clearly, if a dishonest user can construct credentials by himself, there is no need for him to collude with other users in order to forge one. In the model, this is simply reflected by the fact that the adversary is more restricted in his choice of the credential type in the (second phase of the) second game than he is in the (second phase of the) third. A system that offers non-transferability also offers unforgeability.

This relationship between unforgeability and non-transferability motivates the following definition of a sound pseudonym system.

DEFINITION 3 A pseudonym system is said to be sound if it offers pseudonym owner protection and credential non-transferability.

As a side comment, note that non-transferability of credentials is probably the most challenging property for a pseudonym system to achieve. How can colluding users be prevented from sharing their credentials? Certainly, if two users share all their secrets, then they can act as each other in all circumstances. Thus, one will always have to assume that users will not share all their secrets, either because they will be prevented by some means, e.g. by the use of tamper-resistant hardware, or because they will be given a sufficiently strong incentive not to. Examples of schemes that follow the latter strategy include the ones in [Lysyanskaya et al., 2000], where sharing credentials implies sharing a highly valued key (this is called 'PKI-assured non-transferability'), and [Camenisch and Lysyanskaya, 2001], where sharing one credential implies sharing all credentials (this is called 'all-or-nothing non-transferability').

\subsection{Unlinkability of pseudonyms}

We now define Game 4 in order to capture the first privacy property required of pseudonym systems, i.e. the property of pseudonym unlinkability. A second (weaker) privacy property is defined in section 2.6.

In the first phase of the Game $4, \mathcal{A}$ is allowed to issue queries from the following set of query types, which are similar but not identical to the first three query types of section 2.2.

runpeprot $(o): \mathcal{A}$ may arbitrarily select an organisation $o \in(I \cup$ $V)$ and issue this query. When this happens, the Challenger selects a user $u$ according to a probability distribution $\mathcal{D}$ from $U$ and makes $u$ and $o$ execute peprot $u, o, p$. He replies true if the protocol execution is successful and false otherwise. (If the execution is successful, $\mathcal{A}$ knows 
that $u$ and $o$ have established a new pseudonym $p \in P$ but learns neither $p$ nor the identity of its owner.)

runciprot $(p, i, t): \mathcal{A}$ may arbitrarily select a pseudonym/issuer pair $(p, i)$

$\in P \times I$ and a credential type $t \in T_{i}$ and issue this query. When this happens, the Challenger selects the owner of $p$ and makes him execute ciprot $_{i, p, t}$ with $i$. He replies true if the protocol execution is successful and false otherwise (including the case where $p$ has no owner). Note that $\mathcal{A}$ does not learn who the owner of $p$ is.

runcsprot $(p, v, t): \mathcal{A}$ may arbitrarily select a pseudonym/verifier pair $(p$,

$v) \in P \times V$ and a credential type $t \in T$ and issue this query. When this happens, the Challenger selects the owner of $p$ and makes him execute $\operatorname{csprot}_{v, p, t}$ with $v$. He replies true if the protocol execution is successful and false otherwise (including the case where $p$ has no owner). Note that $\mathcal{A}$ does not learn who the owner of $p$ is.

corruptUser $(u)$ : As in section 2.2.

corruptIssuer $(i)$ : As in section 2.2.

corruptVerifier $(u)$ : As in section 2.2.

We now describe the second phase of the Game 4. We denote the set of pseudonyms that belong to uncorrupted users by $P^{* *} \stackrel{\text { def }}{=} P^{*}-\bigcup_{u \in \hat{U}} P_{u}$.

GAME 4 (pseudonym unlinkability): $\mathcal{A}$ outputs two distinct pseudonyms $p_{1}, p_{2}$

$\in P^{* *}$. We say that $\mathcal{A}$ wins the game iff $f\left(p_{1}\right)=f\left(p_{2}\right)$.

$\mathcal{A}$ may apply a variety of strategies in his effort to correlate pseudonyms. We now consider what is probably the most naive strategy and arrive at the following simple result.

LEMMA 1 If the Challenger, during runpeprot(o) queries of an instance of Game 4, selects users uniformly at random (i.e. $\mathcal{D}$ is the uniform distribution), and two pseudonyms, $p_{1}, p_{2}$ say, are chosen at random from $P^{* *}$, then the probability that $f\left(p_{1}\right)=f\left(p_{2}\right)$ is $1 /|U-\hat{U}|$.

Proof Suppose $f\left(p_{1}\right)=u \in(U-\hat{U})$. Then the probability that $f\left(p_{2}\right)=u$ is $1 /|U-\hat{U}|$, since the pseudonyms are allocated uniformly at random to users, and hence also to uncorrupted users. The result follows.

Thus it is tempting to define a pseudonym system that offers unlinkability of pseudonyms as a system where $\mathcal{A}$ cannot win the Game 4 with probability greater than $1 /|U-\hat{U}|+\epsilon(k)$ for any negligible function $\epsilon$. However, this is only a reasonable definition of unlinkability if 
$\mathcal{D}$ is the uniform distribution and if no credentials are shown during the first phase of the game, i.e. there are no instances of runcsprot. Any instance of runcsprot potentially provides the adversary with information about possible links between pseudonyms, and hence potentially increases the adversary's probability of success in linking pseudonyms. Thus, the definition of pseudonym unlinkability needs to take this additional information into account.

Assuming a sound pseudonym system, there are two types of deduction that can be made.

- Suppose a runcsprot invocation, say $\operatorname{runcsprot}(p, v, t)$ for some $p, v$ and $t$, issued at time $\tau$, returns true. Then $\mathcal{A}$ can deduce that there exists some $p^{\prime} \in \bigcup_{u \in U} P_{u, t}(\tau)$ such that $f(p)=f\left(p^{\prime}\right)$.

- Suppose a runcsprot invocation, say $\operatorname{runcsprot}(p, v, t)$ for some $p, v$ and $t$, issued at time $\tau$, returns false. Then $\mathcal{A}$ can deduce that $f(p) \neq f\left(p^{\prime}\right)$ for all $p^{\prime} \in \bigcup_{u \in U} P_{u, t}(\tau)$.

In any instance of Game 4, which in its first phase will involve a series of queries, $\mathcal{A}$ will be able to make a series of deductions about matchings of pseudonyms based on the outcomes (\{true,false $\}$ ) of runcsprot queries (as above). As a result, for each pair of distinct pseudonyms $p_{1}, p_{2} \in P^{* *}, \mathcal{A}$ will be able to compute the probability $P_{p_{1}, p_{2}}$ that $f\left(p_{1}\right)=f\left(p_{2}\right)$ based on these observations (assuming that $\mathcal{A}$ makes optimal use of the information provided). $\mathcal{A}$ also takes into account the probability distribution $\mathcal{D}$ used by the Challenger to select the user during runpeprot queries.

We now define $\bar{P}$ to be the maximum of these probabilities, i.e.

$$
\bar{P} \stackrel{\text { def }}{=} \max _{\substack{p_{1}, p_{2} \in P^{* *} \\ p_{1} \neq p_{2}}}\left(P_{p_{1}, p_{2}}\right) \text {. }
$$

We can now define the notion of pseudonym unlinkability.

DEFINITION 4 A sound pseudonym system is said to offer pseudonym unlinkability iff no $\mathcal{A}$ can win Game 4 with probability greater than $\bar{P}+$ $\epsilon(k)$ for any negligible function $\epsilon$.

An example scenario of how the two types of deduction might be applied in order to calculate $\bar{P}$, is given in the Appendix.

\subsection{Discussion}

In real life, colluding organisations could come up with many more effective strategies in order to correlate pseudonyms. Examples include 
attacks that take into account information such as the time or the geographical location of events that occur in the system. These attacks, however, are not captured by the model, simply because they lie at a different level of abstraction. Protection against, say, timing attacks, de-anonymising traffic analysis or social engineering, is required irrespectively of which particular pseudonym system is being used. The only adversarial strategies to correlate pseudonyms that are inherent in the system, and therefore lie at the same level of abstraction, are the following.

1 If some user is asked for but fails to produce a credential of a given type, the colluding organisations know that none of the pseudonyms on which a credential of that type was previously issued belongs to that user.

2 If some user successfully shows a credential of a given type on one of his pseudonyms, the colluding organisations know that at least one of the pseudonyms on which a credential of that type was previously issued belongs to that user.

These strategies are captured by the probability bound $\bar{P}$. A pseudonym system cannot protect against these strategies without breaching one of its essential properties: that of credential non-transferability. In other words, if a (sound) pseudonym system satisfies Definition 4, this means that the probability that pseudonyms can be successfully linked does not exceed the given bound (by a non-negligible quantity), provided that no 'out-of-scope' attacks place.

\subsection{Indistinguishability of pseudonyms}

We now establish our second privacy property, namely the notion of indistinguishability of pseudonyms and show that it is a necessary condition for pseudonym unlinkability.

Consider the following game between a Challenger and a polynomial time (and space) adversary $\mathcal{A}$. First, the Challenger chooses a sound pseudonym system and a security parameter $k$. On input $k$, he runs init and gives the set $U$ of users to $\mathcal{A}$. $\mathcal{A}$ then chooses two users $u_{0}, u_{1} \in U$ and gives them to the Challenger. The Challenger now flips an unbiased random bit $b \in\{0,1\}$ and makes $u_{b}$ execute peprot ${ }_{u, o, p}$ with some organisation $o \in(I \cup V)$. He then gives $o$ 's private information (including the protocol view and the resulting pseudonym $p$ ) to $\mathcal{A}$.

GAME 5 (pseudonym indistinguishability): $\mathcal{A}$ outputs a bit $b^{\prime} \in\{0,1\}$. We say that $\mathcal{A}$ wins the game iff $b^{\prime}=b$ with probability $\operatorname{Pr}>1 / 2+\epsilon(k)$, for any negligible function $\epsilon$. 
DEFINITION 5 A pseudonym system is said to offer indistinguishability of pseudonyms iff no adversary $\mathcal{A}$ can win the above game.

THEOREM 1 If a sound pseudonym system offers pseudonym unlinkability it also offers pseudonym indistinguishability.

Proof Suppose the converse, i.e. suppose the pseudonym system offers pseudonym unlinkability but does not offer pseudonym indistinguishability. Given $\mathcal{A}^{1}$, an adversary that breaks pseudonym indistinguishability, we construct $\mathcal{A}^{\mathbf{u}}$, an adversary that breaks pseudonym unlinkability, as follows. While playing Game 4 (unlinkability) with the Challenger, $\mathcal{A}^{\mathrm{u}}$ plays the role of the Challenger in Game 5 (indistinguishability) with $\mathcal{A}^{\mathbf{i}}$.

Choose a negligible function $\epsilon$. Let $\mu(k)=\sqrt{\epsilon(k) / 2}$, which, by definition, is also negligible. In Game $4, \mathcal{A}^{\mathrm{u}}$ corrupts all but two users, say $u_{0}$ and $u_{1}$, and one organisation, say o, i.e. $(U-\hat{U})=\left\{u_{0}, u_{1}\right\}$ and $(I-\hat{I}) \cup(V-\hat{V})=\{o\}$. Then $\mathcal{A}^{\mathfrak{u}}$ issues runpeprot $(o)$ queries until three pseudonyms, say $p_{1}, p_{2}$ and $p_{3}$, are established between $o$ and $\left\{u_{0}, u_{1}\right\}$. $\mathcal{A}^{\mathrm{u}}$ does not issue any runcsprot queries.

$\mathcal{A}^{\mathrm{u}}$ then plays three instances of Game 5 (indistinguishability) with $\mathcal{A}^{1}$; in all these games he gives the set of users $U=\left\{u_{0}, u_{1}\right\}$ to $\mathcal{A}^{1}$. In the first he gives the pseudonym $p_{1}$ to $\mathcal{A}^{\mathbf{i}}$, in the second $p_{2}$, and in the third $p_{3}$ (together with $o$ 's private information and corresponding peprot views). Denote $\mathcal{A}^{1}$ 's output occurring in the three instances of Game 5 by $b_{1}, b_{2}$ and $b_{3}$ respectively. Now, since we have assumed that $\mathcal{A}^{\mathfrak{1}}$ breaks pseudonym indistinguishability, we suppose that $\mathcal{A}^{\mathbb{1}}$ wins all instances of Game 5 with probability $1 / 2+\delta(k)$, where $\delta(k)>\mu(k)$ for all sufficiently large $k$.

$\mathcal{A}^{\mathrm{u}}$ now selects $j, j^{\prime} \in\{1,2,3\}, j \neq j^{\prime}$, such that $b_{j}=b_{j^{\prime}}$, where the pair $\left(j, j^{\prime}\right)$ exists by the pigeonhole principle, and outputs $\left(p_{j}, p_{j^{\prime}}\right)$. Now, since $b_{j}=b_{j^{\prime}}$ and $f\left(p_{j}\right), f\left(p_{j^{\prime}}\right) \in\left\{u_{0}, u_{1}\right\}$, we know that $f\left(p_{j}\right)=f\left(p_{j^{\prime}}\right)$ if either $\left(f\left(p_{j}\right)=u_{b_{j}}\right.$ and $\left.f\left(p_{j^{\prime}}\right)=u_{b_{j}}\right)$ or $\left(f\left(p_{j}\right) \neq u_{b_{j}}\right.$ and $\left.f\left(p_{j^{\prime}}\right) \neq u_{b_{j}}\right)$. Hence:

$$
\begin{aligned}
\operatorname{Pr}\left(f\left(p_{j}\right)=f\left(p_{j^{\prime}}\right)\right)= & \operatorname{Pr}\left(f\left(p_{j}\right)=u_{b_{j}}\right) \cdot \operatorname{Pr}\left(f\left(p_{j^{\prime}}\right)=u_{b_{j}}\right) \\
& +\operatorname{Pr}\left(f\left(p_{j}\right) \neq u_{b_{j}}\right) \cdot \operatorname{Pr}\left(f\left(p_{j^{\prime}}\right) \neq u_{b_{j}}\right) \\
= & (1 / 2+\delta(k))^{2}+(1 / 2-\delta(k))^{2} \\
= & 1 / 2+2 \delta(k)^{2} \\
> & 1 / 2+2 \mu(k)^{2} \text { (for all sufficiently large } k \text { ) } \\
= & 1 / 2+\epsilon(k)
\end{aligned}
$$

where $\epsilon$ was assumed to be negligible. Thus $\mathcal{A}^{u}$ breaks unlinkability, contradicting our assumption, and the result follows. 


\subsection{Anonymity of users}

Consider a sound pseudonym system that offers pseudonym unlinkability. The owner $u \in(U-\hat{U})$ of pseudonym $p(u=f(p))$ is hidden in the anonymity set $U-\hat{U}$ because, from $\mathcal{A}$ 's point of view, any user in that set could potentially be the owner of $p$. The effective size of the anonymity set, however, depends on the probability distribution $\mathcal{D}$ according to which users are selected during pseudonym establishment. Using the information-theoretic anonymity metric of [Serjantov and Danezis, 2002; Steinbrecher and Koepsell, 2003], this is given by $-\Sigma_{p \in P^{* *}} \operatorname{Pr}(f(p)=u) \log _{2}[\operatorname{Pr}(f(p)=u)]$ and is maximised if $\mathcal{D}$ is the uniform distribution. In this case the effective size of the anonymity set for all pseudonyms is $\log _{2}|U-\hat{U}|$. It is worth observing that, in the general case, it makes sense to consider the anonymity of the user while acting using a particular pseudonym. In other words, it is likely that the anonymity a user enjoys will depend on the pseudonym under which he is acting.

The above measure of anonymity only applies to a naive adversary; it only takes into account the a priori knowledge (i.e. the distribution $\mathcal{D})$. After observing the system for some time, in the sense of Game 4, $\mathcal{A}$ may decrease the unlinkability between pseudonyms. This decrease in unlinkability yields an a posteriori probability distribution $\mathcal{D}^{\prime}$, that $\mathcal{A}$ is able to construct using deductions that he can make due to the scheme's soundness. While it is the distribution $\mathcal{D}^{\prime}$ that defines the (effective) size of the anonymity set in which users are hidden (while acting under one of their pseudonyms), this does not necessarily mean that a reduction in unlinkability implies a reduction in anonymity in the theoretical definition of the term. Of course, in practice, any linking of pseudonyms is likely to lead to an increased risk of loss of anonymity because of 'out of scope' attacks. As a result, unlinkability is a property of great importance in its own right.

\section{FUTURE WORK AND CONCLUDING REMARKS}

In this paper we have introduced a complexity theoretic model for anonymous credential systems. We have formally defined the notions of pseudonym owner protection, credential unforgeability, credential nontransferability and pseudonym unlinkability. A key challenge is thus to construct scheme(s) that meet the definitions in this model, and/or to prove, under appropriate assumptions, the security of existing ones. There is, however, room to refine and extend the model itself; determining the probability $\bar{P}$ by which colluding organisations should be bound 
when trying to correlate pseudonyms, given a specific history of events in the system, is clearly of importance. Naive strategies for computing $\bar{P}$ appear to be of exponential complexity. Hence, incorporating efficient strategies for computing, approximating or bounding $\bar{P}$ into the model is a desirable refinement. It is envisaged that a refined version of the model described above will combine complexity theory and probability theory in order to describe the resulting degrees of unlinkability and anonymity using recently proposed information theoretic metrics [Serjantov and Danezis, 2002; Steinbrecher and Koepsell, 2003]. This should provide further insight into the inherent limits of unlinkability and anonymity in credential systems. We believe that this will also provide insight as to what they have to achieve in order not to be considered the weakest link with respect to the overall system of which they form part. An extended version of the model could capture additional properties of pseudonym systems, for example credentials that can be shown only a limited number of times and anonymity revocation.

Another direction for future research is the analysis of real-world distributions $\mathcal{D}$ of pseudonym-to-user mappings. This might lead to the description of strategies that users might follow, in a realistic setting, in order to maximise the unlinkability of their pseudonyms. Given the statistical properties of the context, this could also lead to descriptions of how long any given pseudonym can be kept before it should be renewed (if the context allows for this).

\section{Acknowledgments}

We would like to thank Sattam Al-Riyami, Alex Dent, Caroline Kudla and Kenny Paterson for their helpful comments on earlier versions of this paper.

\section{Appendix: An Example}

The following example scenario illustrates how the adversarial strategies are captured by the probability bound $\bar{P}$. For the sake of simplicity, in the example are only one issuer which issues only two types of credential, one verifier and three users. It is assumed that, during the first phase of Game 4 (unlinkability), the adversary corrupts all parties except for the three users, i.e. $I=\hat{I}=\{i\}, V=\hat{V}=\{v\}, U=\left\{u_{1}, u_{2}, u_{3}\right\}$, $\hat{U}=\emptyset$ and $T^{*}=T_{i}=\left\{t_{1}, t_{2}\right\}$.

Table A.1 depicts the queries that $\mathcal{A}$ issues in this example scenario. From the first runcsprot query, $\mathcal{A}$ can deduce that $f\left(p_{4}\right)=f\left(p_{1}\right)$ or $f\left(p_{4}\right)=f\left(p_{2}\right)$ or $f\left(p_{4}\right)=$ $f\left(p_{3}\right)$. From the second runcsprot query, $\mathcal{A}$ can deduce that $f\left(p_{5}\right) \neq f\left(p_{1}\right)$ and $f\left(p_{5}\right) \neq f\left(p_{2}\right)$ and $f\left(p_{5}\right) \neq f\left(p_{3}\right)$. From the third runcsprot query, $\mathcal{A}$ can deduce that $f\left(p_{6}\right) \neq f\left(p_{1}\right)$ and $f\left(p_{6}\right) \neq f\left(p_{2}\right)$ and $f\left(p_{6}\right) \neq f\left(p_{3}\right)$.

Combining the three runcsprot queries, $\mathcal{A}$ can deduce, with certainty, that $f\left(p_{4}\right) \neq$ $f\left(p_{5}\right)$ and that $f\left(p_{4}\right) \neq f\left(p_{6}\right)$. It follows that $p_{5}$ and $p_{6}$ must belong to the two 
Table A.1. Example scenario: runpeprot queries that returned true.

\begin{tabular}{llllll}
\hline Time & Query type & Org & Pseudonym & Type & Outcome \\
\hline 1 & runpeprot & $i$ & $p_{1}$ & n/a & true \\
2 & runpeprot & $i$ & $p_{2}$ & n/a & true \\
3 & runpeprot & $i$ & $p_{3}$ & n/a & true \\
4 & runpeprot & $v$ & $p_{4}$ & n/a & true \\
5 & runpeprot & $v$ & $p_{5}$ & n/a & true \\
& & & & & \\
6 & runciprot & $i$ & $p_{1}$ & $t_{1}$ & true \\
7 & runciprot & $i$ & $p_{1}$ & $t_{2}$ & true \\
8 & runciprot & $i$ & $p_{2}$ & $t_{1}$ & true \\
9 & runciprot & $i$ & $p_{2}$ & $t_{2}$ & true \\
10 & runciprot & $i$ & $p_{3}$ & $t_{1}$ & true \\
& & & & & \\
11 & runcsprot & $i$ & $p_{4}$ & $t_{1}$ & true \\
12 & runcsprot & $i$ & $p_{5}$ & $t_{1}$ & false \\
13 & runcsprot & $i$ & $p_{6}$ & $t_{1}$ & false \\
\hline
\end{tabular}

users $\left\{u_{1}, u_{2}, u_{3}\right\}-\left\{f\left(p_{4}\right)\right\}$. So, the probability $P_{p_{5}, p_{6}}$ that $f\left(p_{5}\right)=f\left(p_{6}\right)$ is $1 / 2$. This happens to be the maximum over all distinct pseudonym pairs and thus, in the example, $\bar{P}=1 / 2$. In other words, if $\mathcal{A}$, at the end of the game, outputs $\left(p_{5}, p_{6}\right)$, he has a 50\% chance of winning the game. If a (sound) pseudonym system offers pseudonym unlinkability, then no $\mathcal{A}$ should be able to break this bound by a nonnegligible quantity.

\section{Notes}

1. In certain existing pseudonym systems, credential types are identified with some form of public verification key. These keys are typically published.

2. This is easily achieved by having a unique identifier of each $i$ embedded into all its types $T_{i}$.

3. We do not specify the probability distribution according to which the Challenger selects $p$ from the set of pseudonyms $u$ has established, since this should not affect security.

4. This requirement is a technicality that we need in order to define the function $f$. It practice it can be met by having peprot select pseudonyms uniformly at random from a large enough set $P$. The pseudonym establishment protocols of some existing schemes are of this form.

\section{References}

Bellare, M. and Rogaway, P. (1994). Entity authentication and key distribution. In Stinson, D., editor, Advances in Cryptology - Crypto 93 Proceedings, volume 773 of Lecture Notes in Computer Science, pages 232-249.

Brands, S. (2000). Rethinking Public Key Infrastructures and Digital Certificates Building in Privacy. The MIT Press, Cambridge, Massachusetts. 
Camenisch, J. and Lysyanskaya, A. (2001). An efficient system for non-transferable anonymous credentials with optional anonymity revocation. In Pfitzmann, B., editor, Advances in Cryptology - EUROCRYPT 2001, International Conference on the Theory and Application of Cryptographic Techniques, Innsbruck, Austria, May 6-10, 2001, Proceedings, volume 2045 of Lecture Notes in Computer Science, pages 93-118. Springer Verlag, Berlin.

Chaum, D. (1985). Security without identification: Transaction systems to make big brother obsolete. In Communications of the ACM, volume 28, pages 1030-1044.

Chaum, D. and Evertse, J.-H. (1987). A secure and privacy-protecting protocol for transmitting personal information between organizations. In Odlyzko, A. M., editor, Advances in Cryptology - CRYPTO '86, Santa Barbara, California, USA, 1986, Proceedings, number 263 in Lecture Notes in Computer Science, pages 118167. Springer Verlag, Berlin.

Damgard, I. (1990). Payment systems and credential mechanisms with provable security against abuse by individuals. In Goldwasser, S., editor, Advances in Cryptology - CRYPTO '88: Proceedings, number 403 in Lecture Notes in Computer Science, pages 328-335. Springer Verlag.

Lysyanskaya, A., Rivest, R. L., Sahai, A., and Wolf, S. (2000). Pseudonym systems. In Heys, H. M. and Adams, C. M., editors, Selected Areas in Cryptography, 6th Annual International Workshop, SAC'99, Kingston, Ontario, Canada, August 910, 1999, Proceedings, volume 1758 of Lecture Notes in Computer Science, pages 184-199. Springer Verlag, Berlin.

Serjantov, A. and Danezis, G. (2002). Towards an information theoretic metric for anonymity. In Dingledine, R. and Syverson, P. F., editors, Privacy Enhancing Technologies, Second International Workshop, PET 2002, San Francisco, CA, USA, April 14-15, 2002, Revised Papers, volume 2482 of Lecture Notes in Computer Science, pages 41-53. Springer-Verlag, Berlin.

Steinbrecher, S. and Koepsell, S. (2003). Modelling unlinkability. In Dingledine, R., editor, Privacy Enhancing Technologies, Third International Workshop, PET 2003, Dresden, Germany, March 26-28, 2003, Revised Papers, volume 2760 of Lecture Notes in Computer Science, pages 32-47. Springer-Verlag, Berlin. 